\title{
BMJ Open Safety and efficacy of self-administered inhaled loxapine (ADASUVE) in agitated patients outside the hospital setting: protocol for a phase IV, single- arm, open-label trial
}

Emilio Gil, Fernando Garcia- Alonso, Anca Boldeanu, Thaïs Baleeiro Teixeira, On behalf of Loxapine Inhaled Home Use study investigator's team

To cite: Gil E, Garcia- Alonso F, Boldeanu A, et al. Safety and efficacy of self-administered inhaled loxapine (ADASUVE) in agitated patients outside the hospital setting: protocol for a phase IV, single-arm, open-label trial. BMJ Open 2018;8:e020242. doi:10.1136/ bmjopen-2017-020242

- Prepublication history and additional material for this paper are available online. To view these files, please visit the journal online (http://dx.doi org/10.1136/bmjopen-2017020242).

Received 27 0ctober 2017 Revised 26 January 2018 Accepted 1 March 2018

Check for updates

(C) Author(s) (or their employer(s)) 2018. Re-use permitted under CC BY-NC. No commercial re-use. See rights and permissions. Published by BMJ.

Medical Department, Ferrer Internacional, S.A., Barcelona, Spain

Correspondence to

Dr Emilio Gil; egil@ferrer.com

\section{ABSTRACT}

Introduction There is a need for fast-acting, non-injection antiagitation treatments that are well tolerated and can be used outside of healthcare facilities. In phase II/III trials, an inhaled formulation of loxapine (ADASUVE®), a wellestablished, first-generation antipsychotic agent, provided rapid control of mild to moderate agitation in the hospital setting. The present study was designed to investigate the safety and efficacy of inhaled loxapine when selfadministered outside the hospital setting.

Methods and analysis This phase IV, multicentre, singlearm, open-label clinical trial is being conducted in five countries in Europe: Spain, Germany, Norway, Romania and Austria. The aim is to include approximately 500 patients with schizophrenia or bipolar disorder who previously received and responded well to inhaled loxapine in the hospital setting. Eligible patients will be followed up for 6 months from baseline. They will be given a $10 \mathrm{mg}$ dose of inhaled loxapine to self-administer outside the hospital setting to treat an agitation episode, should one occur. Patients will also be given a short-acting beta-agonist bronchodilator for treatment of possible severe respiratory side effects. The primary endpoint is incidence of serious adverse events (AEs) and respiratory AEs of special interest related to use of inhaled loxapine outside the hospital setting. Secondary endpoints include incidence of other AEs, Clinical Global Impression-Improvement scores up to 2 hours after self-administration of inhaled loxapine, time to improvement of agitation, patient satisfaction with treatment, treatment outcomes according to agitation severity and concordance between the patient (or a family member/caregiver) and the physician in scoring of agitation severity and the decision to self-administer inhaled loxapine.

Ethics and dissemination The protocol received ethics committee approval in the participating countries between January and August 2016. The results of this study will be disseminated through one or more scientific papers. Trial registration number EudraCT2015-003331-36; NCT02525991; Pre-results.

\section{INTRODUCTION}

Agitation is a serious medical challenge in many patients with schizophrenia ${ }^{1}$ or bipolar

\section{Strengths and limitations of this study}

- Relevant sample size from different European countries.

- Inhaled loxapine is a self-administered non-invasive treatment.

- Use of a subjective scale (Clinical Global Impression (CGI)) to assess the effectiveness of treatment.

- Patients trained on identification of an agitation crisis and use of the CGI scale.

Possible severe respiratory side effects.

disorder. $^{2}$ Symptoms include excitement, physical and verbal hyperactivity, hostility, tension and threatening behaviour, ${ }^{3}$ and patients may need to be physically restrained or secluded for their own protection and that of the people around them.

Agitation associated with psychosis is a frequent reason for emergency department visits, admission to a psychiatric in-patient ward and prolongation of hospitalisation. ${ }^{4}$ Because it can escalate very rapidly, ${ }^{6}$ agitation needs to be recognised and treated early if an optimal outcome is to be achieved. Also, if escalation of agitation can be prevented, emergency department visits could be avoided, saving public healthcare both money and resources. ${ }^{7}$

The prevalence of schizophrenia in adults has been estimated at approximately $1 \%$ in the $\mathrm{USA}^{8}$ and Europe. ${ }^{9}{ }^{10}$ Worldwide, more than 21 million people are thought to have schizophrenia. ${ }^{11}$ Current treatments are symptomatic, with antipsychotics typically providing adequate relief. However, factors such as non-adherence to treatment mean that symptoms often recur or worsen.

Bipolar disorder affects more than 30 million people worldwide, and is among 
the top 20 leading causes of disability. ${ }^{12} \mathrm{Up}$ to $2 \%$ of Europeans will have bipolar disorder at some point in their life, with approximately half of them developing bipolar I disorder. ${ }^{13} 14$

Episodes of agitation are typically treated with antipsychotic drugs, which are available in a variety of forms, including oral tablets and intramuscular (IM) injections. Time to onset is an important factor when selecting an appropriate antiagitation treatment. ${ }^{15}$ IM formulations provide faster relief compared with oral formulations. ${ }^{61617}$ Nevertheless, the delay in onset of action of IM formulations is often sufficient for symptoms to escalate. ${ }^{1617}$ Moreover, unwillingness to receive an IM injection can exacerbate agitation, increasing risks to both patients and caregivers (such as the risk of physical injury during restraint). Other factors limiting the use of IM injections include trauma to the patient that compromises the patient-physician relationship and problems with longterm compliance. ${ }^{15}$ Intravenous administration, which would produce rapid effects, is generally not feasible in severely agitated patients.

Loxapine is a first-generation antipsychotic agent belonging to the dibenzoxazepine class. It is used to treat schizophrenia ${ }^{1819}$ and has shown effectiveness as an antiagitation treatment when injected intramuscularly. ${ }^{20}$ Like other antipsychotic drugs, the clinical effects of loxapine are likely due to its antagonism of dopamine D2 receptors. $^{18}$

There is an acute need for self-administered drugs that provide rapid symptom relief and are easy to use. Loxapine has been reformulated into an inhalable powder (ADASUVE®) and its one-breath technology allows direct administration of the drug to the lungs, resulting in rapid absorption into the systemic circulation. Inhaled loxapine was developed for the treatment of agitation in patients with schizophrenia or bipolar I disorder and has received regulatory approval from the US Food and Drug Administration (FDA) and the European Medicines Agency (EMA) for use in adults.

The FDA and the EMA both require that inhaled loxapine only be used in healthcare facilities under restricted conditions. ${ }^{21}$ Phase II/III clinical trials in patients with schizophrenia ${ }^{22} 23$ or bipolar I disorder ${ }^{24}$ found that administration of inhaled loxapine in the hospital setting significantly reduced agitation (scored using the Positive and Negative Syndrome Scale-Excited Component) in 2 hours compared with placebo. Moreover, a significant decrease in agitation versus placebo was observed after only $10 \mathrm{~min}$. Thus, inhaled loxapine appears to rapidly reduce agitation in patients with schizophrenia or bipolar I disorder.

Rapid-acting antiagitation treatments that are well tolerated and easy to administer outside of healthcare facilities remain unavailable. Such treatments would empower patients to treat themselves without being supervised by a healthcare professional. Importantly, previous research suggests the decision-making ability of patients with schizophrenia is not impaired. ${ }^{25}$ However, with unsupervised self-treatment, there is a risk of patients treating themselves unnecessarily (ie, when they are not agitated) or taking too many or too high doses of a particular treatment. One way to safeguard against such misuse might be for a patient's caregiver or family member to assist with self-treatment.

The present study was designed to investigate whether inhaled loxapine can be used as a non-invasive treatment to provide rapid control of mild to moderate agitation when administered in hospitals or at home. The primary objective is to assess the safety profile of self-administered inhaled loxapine outside the hospital setting in patients who are known to respond to inhaled loxapine and are well trained in its use. The primary focus is on serious adverse events (SAEs) and respiratory AEs of special interest (AESI) related to inhaled loxapine. Secondary objectives are to evaluate the following after self-administration of inhaled loxapine outside the hospital setting: Clinical Global Impression-Improvement (CGI-I) up to 2 hours after treatment, as scored by the patient or a family member/caregiver; time to improvement of agitation; patient satisfaction with treatment; treatment outcomes according to agitation severity; and concordance between the patient (or family member/caregiver) and the physician in scoring of agitation severity and the decision to self-administer inhaled loxapine.

\section{METHODS AND ANALYSIS \\ Study design}

This is an ongoing phase IV, multicentre, single-arm, open-label clinical trial conducted in five countries in Europe (Spain, Germany, Norway, Romania and Austria) to evaluate the safety of inhaled loxapine when self-administered by agitated patients outside the hospital setting without the supervision of a healthcare professional. The study design is summarised in figure 1.

\section{Study population}

The study population will comprise approximately 500 adult patients with schizophrenia or bipolar disorder. Consecutive patients attending a hospital setting (eg, a hospital ward, emergency room or inpatient unit) for treatment of an agitation episode will be screened for eligibility to participate in the study. Staff at the study sites will also screen consecutive patients who have had an agitation episode during the previous 6 months.

Eligible patients are those treated with inhaled loxapine before or during screening and identified as responders, defined by (1) a CGI-I score at 2 hours after inhaled loxapine administration of either 1 (Very much improved) or 2 (Much improved) or (2) an equivalent clinical evaluation at the discretion of the investigator. Participants must fulfil all of the inclusion criteria and none of the exclusion criteria shown in table 1 . 


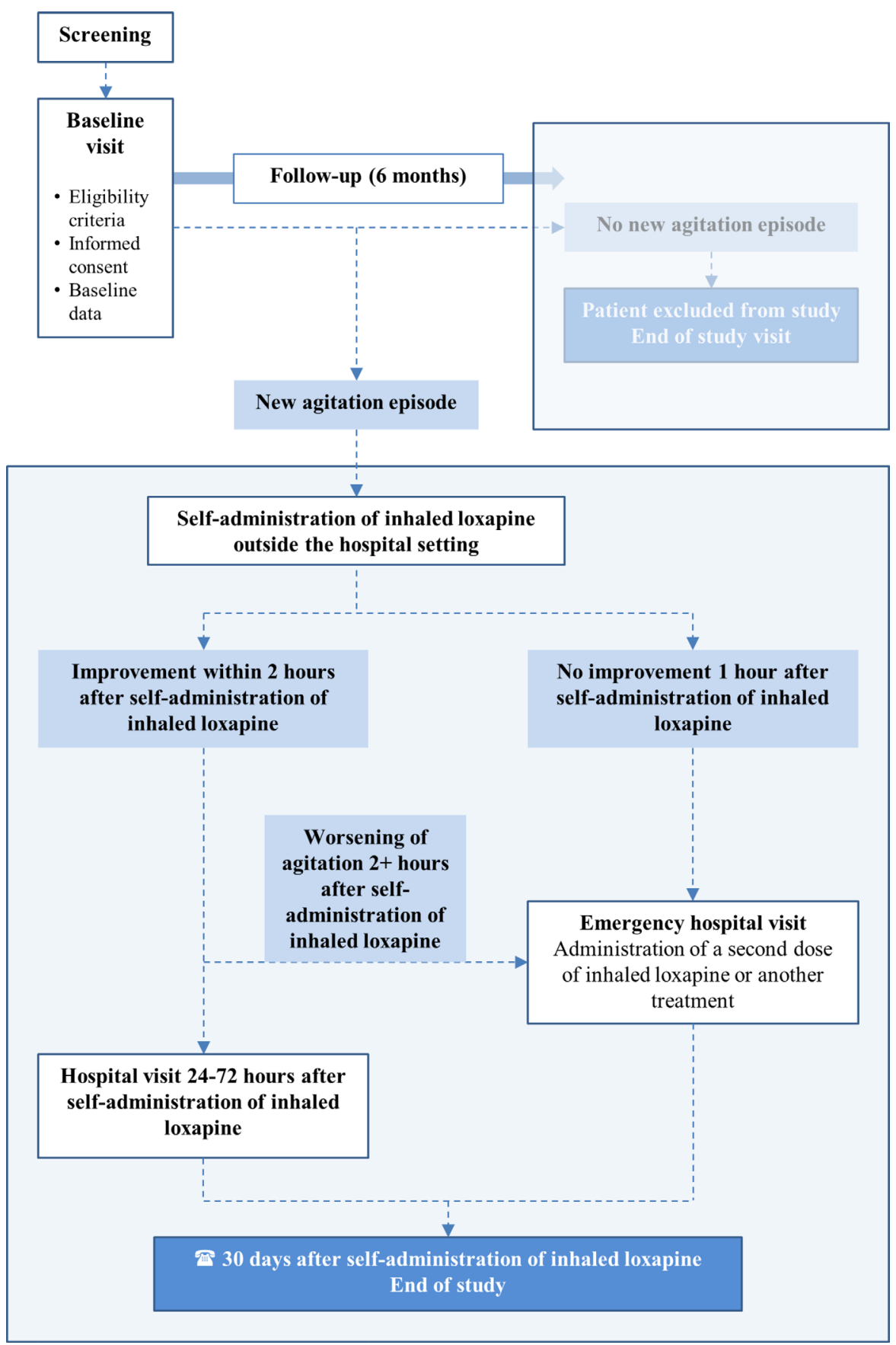

Figure 1 Overall design of the study.

\section{Intervention}

Within 4 weeks after screening, each eligible patient will have a baseline hospital visit. Each patient will be given (1) a single-dose inhaler containing $10 \mathrm{mg}$ of loxapine (and delivering $9.1 \mathrm{mg}$ of loxapine) and (2) a short-acting beta-agonist bronchodilator (salbutamol) as rescue medication for possible severe respiratory side effects (bronchospasm). They will also be given instructions on how to store and use inhaled loxapine, and how to identify an agitation crisis by recognising the escalating symptoms experienced in previous agitation episodes. Because patients will only be given a single dose of inhaled loxapine to self-administer, it will not be possible for them to take too many doses.

For patients who self-administer inhaled loxapine outside the hospital setting to treat an acute agitation episode, there are three clinical scenarios:

1 . If there is an improvement within 2 hours after self-administration of inhaled loxapine, a follow-up hospital visit with the study investigator will be scheduled for 24-72 hours after self-administration of inhaled loxapine. The study investigator will confirm that the patient is in good general health, assess the severity of the agitation episode before self-administration of inhaled 
Table 1 Eligibility criteria

\section{Inclusion criteria}

1 Male and female patients aged 18-65years

2 Patient (or legal representative) willing and able to provide written informed consent

\section{Exclusion criteria}

Diagnosis of dementia

Serious and unstable illnesses, including current hepatic, renal, gastroenterological, respiratory, cardiovascular (including ischaemic heart disease and congestive heart failure), endocrinological, neurological (including stroke, transient ischaemic attack, subarachnoid bleeding, brain tumour, encephalopathy and meningitis) disease

3 Diagnosis of schizophrenia or bipolar disorder (according to DSM- History of allergic reaction to loxapine or amoxapine IV-TR, DSM-V or ICD-10 criteria)

4 Ongoing agitation episode (mild or moderate) or previous agitation Use of an investigational drug within 30 days before episode within the 6 months prior to screening managed in the hospital setting

5 Previous treatment with inhaled loxapine with a positive outcome according to the CGI-I scale (CGI-I score of 1 or 2 at 2 hours after administration of inhaled loxapine), or an equivalent clinical evaluation at the discretion of the investigator, and no bronchospasm after previous administration of inhaled loxapine

6 No active respiratory disease such as acute respiratory signs/ symptoms

(eg, wheezing) or active airway disease (asthma, chronic obstructive pulmonary disease or emphysema)

7 Patient and/or family member/caregiver able to:

a. Understand and follow specific training for the administration of inhaled loxapine outside the hospital setting

b. Identify/detect a respiratory problem

c. Use of a bronchodilator if necessary

8 A family member or other caregiver who:
a. Is male or female
b. Is $\leq 80$ years old
c. Can spend 3+ consecutive hours with the patient
d. Has good physical and psychological health, without physical
limitations
e. Is able to read and write
f. Is able to understand and follow the study procedures

9 Medical record data available for the patient's previous treatment with inhaled loxapine in the hospital setting

10 For women of childbearing potential (not including females who are surgically sterile or postmenopausal with no menstruation during the previous 24

months), confirmation that they:

a. Are non-lactating

b. Are non-pregnant (negative pregnancy test result at baseline)

c. Agree to use a medically acceptable and effective birth control

method throughout the study and for 1 week following the end of the study

First patient in was on 8 September 2016.

Last patient out is predicted to be in September 2018.

CGI-I, Clinical Global Impression-Improvement; DMS-IV-TR, Diagnostic and Statistical Manual of Mental Disorders, Fourth Edition Text Revision; DMS-V, Diagnostic and Statistical Manual of Mental Disorders, Fifth Edition.

loxapine using the CGI-S scale and indicate whether they agree or disagree with the decision to self-administer inhaled loxapine.

2. If there is worsening or no improvement of the agitation episode 1 hour after self-administration of inhaled loxapine, the patient must attend the study hospital to receive a second dose of inhaled loxapine (to be administered 2+ hours after the first dose) or another medication to treat the agitation episode, at the discretion of the investigator. 
3. If initial improvement of the agitation episode within 2 hours after self-administration of inhaled loxapine is followed by worsening of the agitation episode $2+$ hours after self-administration of inhaled loxapine, the patient must attend the study hospital to receive a second dose of inhaled loxapine or another medication to treat the agitation episode, at the discretion of the investigator.

Patients will receive a follow-up call 30 days after self-administration of inhaled loxapine outside the hospital setting to determine their general health status and to obtain information about any other AEs related to inhaled loxapine during the intervening period.

\section{Study procedures}

At the baseline visit, the patient (or their legal representative) will be asked by the investigator or the study staff to provide signed informed consent for inclusion in the study and their eligibility will be confirmed (a copy of the patient consent form used in the study is provided as a supplementary file). The patient will then be provided with inhaled loxapine to treat the next agitation episode outside the hospital setting. Each patient and their family member/caregiver will also be given a diary card in which to record information on agitation episodes.

At baseline, the following will be recorded in an electronic case report form (eCRF): demographics, diagnosis, current/previous agitation episodes, respiratory disease history and risks factors, other comorbidities/medical history and previous inhaled loxapine treatment.

Following the baseline visit, there will be 6 months of follow-up, during which it is expected that a new episode of agitation will occur. Data will only be collected for the first new episode of agitation during follow-up. Approximately every 3 months, the study staff will conduct a 10 min phone call with the enrolled patient or their family member/caregiver to find out about the patient's general health status. Patients who do not have a new episode of agitation during follow-up will be scheduled for an end of study visit and will be excluded from the study.

During the first new episode of agitation outside the hospital setting, the following data will be recorded in the patient's diary card with family member/caregiver support: (1) date/time of onset of the agitation episode, (2) date/time of inhaled loxapine self-administration, (3) severity of the agitation episode (CGI-Severity (CGI-S) score) before and 2, 10, 20, 30, 60 and $120 \mathrm{~min}$ after self-administration of inhaled loxapine and (4) AEs during the 24 hours after inhaled loxapine self-administration. In addition, patients who show improvement of agitation within 2 hours after self-administration of inhaled loxapine will be asked to evaluate their satisfaction with the inhaled loxapine treatment on a fivepoint Likert scale at the follow-up hospital visit with the study investigator 24-72 hours after self-administration of inhaled loxapine. For patients who attend the study hospital because of no improvement or worsening of agitation after self-administration of inhaled loxapine, the following data will be recorded in the eCRF by the study staff: (1) treatments other than inhaled loxapine administered for the agitation episode, (2) date/time of onset of the agitation episode, (3) time to improvement of the agitation episode and (4) AEs related to inhaled loxapine.

\section{Endpoints}

The primary endpoint is incidence of SAEs and respiratory AESI related to self-administration of inhaled loxapine outside the hospital setting. The secondary endpoints are: (1) incidence of non-respiratory AESI and other AEs related to self-administration of inhaled loxapine outside the hospital setting; (2) incidence of SAEs, AESI and other AEs related to the second dose of inhaled loxapine administered at the study hospital; (3) time to improvement of the current episode of agitation; (4) absolute CGI-I scores up to 2 hours after self-administration of inhaled loxapine; (5) percentage of inhaled loxapine responders, calculated as the proportion of patients with a CGI-I score of 1 or 2 at 2 hours after self-administration of inhaled loxapine; (6) patient satisfaction with treatment (inhaled loxapine responders only); (7) antiagitation medications administered at the hospital (including inhaled loxapine) to treat agitation episodes that worsen or do not improve after self-administration of inhaled loxapine; (8) concordance between the patient/family member/caregiver and physician in terms of scoring of agitation severity (CGI-S) before self-administration of inhaled loxapine and the decision to self-administer inhaled loxapine; (9) patient/ family member/caregiver demographics and clinical characteristics of patients who self-administer inhaled loxapine; and (10) differences in demographic/clinical profiles and outcomes after inhaled loxapine treatment according to agitation severity (mild vs moderate) based on the CGI-S scale.

\section{CGI scale}

The CGI scale ${ }^{26}$ was developed to give a brief clinical assessment of a patient's psychiatric disease severity before and after they start taking a study medication. ${ }^{27}$ It is quick to use and can be applied to all psychiatric diseases and all medications. The CGI has two components: CGI-S, used to rate disease severity, and CGI-I, used to rate change in disease severity since the start of treatment (irrespective of whether any change is due to treatment).

Clinical Global Impression-Severity

$1=$ Normal, not at all ill, 2=Borderlinementally ill, 3=Mildlyill, 4=Moderatelyill 5=Markedly ill, $6=$ Severelyill, $7=$ Among the most extremely ill patients

Clinical Global Impression-Improvement

1=Verymuch improved, 2=Much improved, 3=Minimally improved, 4=No change, $5=$ Minimallyworse, $6=$ Much worse, $7=$ =ery much worse

CGI-S and CGI-I will be scored by the patient or their family member/caregiver. 


\section{Patient satisfaction with treatment}

Treatment satisfaction will be evaluated in inhaled loxapine responders at the hospital visit 24-74hours after self-administration of inhaled loxapine using the question 'Are you/ Were you satisfied with your treatment?' Patients will be asked to select one of the following answers: 'Very satisfied', 'Satisfied', 'Uncertain', 'Dissatisfied' or 'Very dissatisfied'. Patients who give an answer of Very satisfied or Satisfied will be considered to be satisfied with the treatment.

\section{Sample size calculation}

The sample size estimation is based on the precisionhalf the width of the CI-for estimating the primary endpoint (incidence of related SAEs and respiratory AESI). In the phase III programme, with respiratory exclusion criteria similar to the contraindications described in the ADASUVE Prescribing Information, ${ }^{28}$ the observed rate of respiratory AESI in persons receiving inhaled loxapine was $0.8 \% .^{23}{ }^{24}$ If the rate of respiratory AESI is slightly higher in this study than in the controlled setting of previous clinical trials, we estimate a respiratory AESI rate of 1\%. A sample size of 500 patients produces a two-sided $95 \%$ CI with a precision of $\pm 0.87 \%$.

\section{Data analysis}

All data analyses will be performed using SASV.9.2 or higher (SAS Institute, Cary, North Carolina, USA) or a comparable software system. The analyses will be based on the safety set, which will comprise all patients who self-administer inhaled loxapine outside the hospital setting. Continuous data will be presented as number of observations, mean, SD, minimum, Q1, median, Q3 and maximum. Data for categorical and ordinal variables will be presented as counts, proportions or percentages with $95 \%$ CIs.

The primary analysis will examine the incidence of SAEs and respiratory AESI related to inhaled loxapine treatment outside the hospital setting. The incidence of an $\mathrm{AE}$ will be defined as the percentage of patients experiencing the AE. 95\% CIs will be calculated for all AE rates. $\mathrm{AE}$ rates will be computed for all study patients combined and also for meaningful subgroups of patients, defined according to diagnosis / cause of agitation (schizophrenia, bipolar disorder, other) and underlying medical conditions (hepatic impairment, renal impairment, cardiovascular disease, suicidal risk).

An important objective of the present study is to determine whether the decision to self-administer inhaled loxapine is in accordance with the clinical judgement of the physician. Kappa index ${ }^{29}$ will be calculated to assess the degree of concordance between the patient/family member/caregiver and physician in terms of (1) scoring of agitation severity (CGI-S) before self-administration of inhaled loxapine and (2) the decision to self-administer inhaled loxapine. The proportion of observations for which the patient/family member/caregiver and physician agree will also be calculated.

\section{ETHICS AND DISSEMINATION}

The protocol received ethics committee approval in Austria on 1 July 2016, at different sites in Germany between 11 January 2016 and 1 August 2016, in Norway on 15 February 2016, in Romania on 26 July 2016 and in Spain on 12 January 2016. The latest version of the protocol includes four amendments (one non-substantial and three substantial), which have received ethics committee approval where applicable. Patient enrolment did not start at any site before written confirmation of approval from the relevant ethics committee.

The study is being conducted in compliance with the protocol, regulatory requirements, International Council of Harmonisation (ICH) E6 Good Clinical Practice and the ethical principles of the latest version of the Declaration of Helsinki, as adopted by the World Medical Association.

The results of this study will be disseminated through one or more scientific papers and may also be presented at medical conferences.

\section{DISCUSSION}

The lack of fast-acting anti-agitation medications that can be self-administered non-invasively has negative consequences in terms of patient quality of life and health system costs. To our knowledge, this is the first study to investigate the self-administration of inhaled loxapine outside the hospital setting. It was initiated to investigate whether self-administration of inhaled loxapine outside the hospital setting might improve the management of patients with agitation.

Recruitment started on 8 September 2016, and by 9 June 2017, 148 patients had been included. Final results are expected in the third quarter of 2018. Data for the first 10 patients to complete the study (five diagnosed with schizophrenia and five with bipolar disorder) are already available. The patients (five men and five women) had a mean (SD) age of 33.1 (9.4) years and a mean (SD) time since diagnosis of 8.5 (7.5) years. No AEs were reported. At $2 \mathrm{~min}$ after self-administration of inhaled loxapine, one patient $(10 \%)$ assessed his/her agitation as Very much improved and two patients (20\%) as Much improved (CGI-I; the other patients reported No change). After $10 \mathrm{~min}$, six patients $(60 \%)$ assessed their agitation as Very much improved, one patient $(10 \%)$ as Much improved and three patients $(30 \%)$ as Minimally improved. Improvements were maintained at 2 hours after self-administration of inhaled loxapine. These preliminary results suggest that inhaled loxapine controlled agitation in the first 10 patients who completed the study and has a good safety profile. Inhaled loxapine may represent a new therapeutic option to achieve rapid control of agitation outside the hospital setting.

This study has some limitations, including the use of a subjective self-rating scale (CGI) to assess the effectiveness of treatment. However, patients and their family members/caregivers are receiving training in use of the CGI scale and the CGI data they provide will be checked 
against the investigator's assessments of (1) severity of the agitation episode before self-administration of inhaled loxapine and (2) whether the decision to self-administer inhaled loxapine was correct. The study's strengths include the inclusion of patients at sites in five European countries, which should mitigate intercountry differences in the management of patients with agitation and increase generalisability of the study findings.

Acknowledgements Medical writing services provided by Stephen Gilliver, PhD, and Neus Valveny, PhD, of TFS were funded by Ferrer Internacional S.A.

Collaborators The following investigators participated in this study: $\mathrm{Dr}$ VicenteTordera, Hospital Lluís Alcanyis; Dr Ramón Palmer Viciedo, Hospital Provincial de Castellón; Dra Purificación Salgado, Hospital del Mar; Dr Jordi Domingo Ribas, Institut NEPP; Dralluminada Corripio Collado, Hospital de la Santa Creu i Sant Pau; DrJosé Manuel Montes, Hospital Ramón y Cajal; Dr Javier Correas Lauffer, Hospital Univ De Henares; Dr Salvador Ruiz Murugarrem, Hospital Príncipe de Asturias; Dr Santiago Ovejero García, Fundación Jiménez Díaz; Dr Fernando Mora, Hospital Infanta Leonor; Dra Presentación Ataz López, Complejo Hospitalario Torrecárdenas; Dr Andres Fontalba Navas, Hospital La Inmaculada; Dra Ana Ma González Pinto, Hospital Santiago Apóstol; Dr RicardoMartínez, Hospital Virgen del Mirón; Dr César García Castrillo, Complejo Hospitalario de Jaen; Dr Francisco Toledo, Hospital Univ Virgen de Arrixaca; Dr Eduard Vieta, Hospital Clínic i Provincial Barcelona; Dr Emilio González, Hermanas Hospitalarias Sociosanitario Palencia Dr Manuel Franco, Complejo Asistencial Zamora; Dra Nicolae Virgil Drasovean, Spitalul de Psihiatrie 'Elisabeta Doamna' Galati; Dr Nils Håvard Dahl, Helse Nord-Trøndelag HV Sykehuset Levanger, Dr Bo Skagen, Spesialistesenteret på Straume-Helsesenteret, Dr David Zilles, Klinik für Psychiatrie und Psychotherapie der UniversitätsmedizinGöttingen; Dr Peter Zwanzger, Allgemeinpsychiatrie und Psychosomatik, Dr Georg Juckel, LWL-Universitätsklinikum Bochum der RuhrUniversität Bochum; Dr Kai G. Kahl, Medizinische Hochschule Hannover; Dr Thomas Messer, Fachklinik für Psychiatrie, Psychotherapie und Psychosomatik; Dr Siegfried Kasper, Medizinische Universität Wien Universitätsklinik für Psychiatrie und Psychotherapie.

Contributors FG-A, EG: substantially contributed to the conception or design of the work, drafted the work and revised it critically for important intellectual content. EG gave the final approval of the version published. AB, TBT: substantially contributed to the conception or design of the work, drafted the work and revised it critically for important intellectual content. TBT gave the final approval of the version published.

Funding This work was supported by Ferrer Internacional S.A.

Competing interests All authors are employees of Ferrer Internacional S.A.

Patient consent Obtained.

Ethics approval Ethics committee approval of the original study protocol was obtained from: CElm Parc de la Salud (Hospital del Mar, Barcelona, Spain; EthikKommission der Medizinische Fakultat, Germany; REK Regionale Komitter for Medisinsk og Helsefaglig Forskningsetikk, Norway; Academia de Stiinte medicale, Comisia Nationala de Bioetica a Medicamentului si a Dispozitivelor Medicale, Romania. Each substantial protocol amendment was granted approval by the relevant ethics committee(s) prior to implementation.

Provenance and peer review Not commissioned; externally peer reviewed.

Open access This is an open access article distributed in accordance with the Creative Commons Attribution Non Commercial (CC BY-NC 4.0) license, which permits others to distribute, remix, adapt, build upon this work non-commercially, and license their derivative works on different terms, provided the original work is properly cited, appropriate credit is given, any changes made indicated, and the use is non-commercial. See: http://creativecommons.org/licenses/by-nc/4.0/.

\section{REFERENCES}

1. Osser DN, Sigadel R. Short-term inpatient pharmacotherapy of schizophrenia. Harv Rev Psychiatry 2001;9:89-104.

2. Alderfer BS, Allen MH. Treatment of agitation in bipolar disorder across the life cycle. J Clin Psychiatry 2003;64:3-9.

3. Battaglia J. The treatment of acute agitation in schizophrenia. CNS Spectr 2007;12:1-16.
4. San L, Marksteiner J, Zwanzger P, et al. State of acute agitation at psychiatric emergencies in europe: the stage study. Clin Pract Epidemiol Ment Health 2016;12:75-86.

5. Pajonk FG, Schmitt P, Biedler A, et al. Psychiatric emergencies in prehospital emergency medical systems: a prospective comparison of two urban settings. Gen Hosp Psychiatry 2008;30:360-6.

6. Garriga M, Pacchiarotti I, Kasper S, et al. Assessment and management of agitation in psychiatry: Expert consensus. World $\mathrm{J}$ Biol Psychiatry 2016;17:86-128.

7. Serrano-Blanco A, Rubio-Valera M, Aznar-Lou I, et al. In-patient costs of agitation and containment in a mental health catchment area. BMC Psychiatry 2017;17:212.

8. National Institute of Mental Health (NIMH). Statistics: Schizophrenia. https://www.nimh.nih.gov/health/statistics/.prevalence/ schizophrenia.shtml.

9. Health \& Consumer Protection Directorate-General. The State of Mental Health in the European Union, 2004. http://www.msssi.gob. es/organizacion/sns/planCalidadSNS/pdf/excelencia/salud_mental/ opsc est18.pdf.pdf.

10. Wittchen HU, Jacobi F, Rehm J, et al. The size and burden of mental disorders and other disorders of the brain in Europe 2010. Eur Neuropsychopharmacol 2011;21:655-79.

11. World Health Organization. Mental health: Schizophrenia. http://www. who.int/mental_health/management/schizophrenia/en/.

12. World Health Organization. The global burden of disease. 2004 http://www.who.int/healthinfo/global_burden_disease/2004_report_ update/en/.

13. Merikangas KR, Jin R, He JP, J-p H, et al. Prevalence and correlates of bipolar spectrum disorder in the world mental health survey initiative. Arch Gen Psychiatry 2011;68:241-51.

14. Pini S, de Queiroz V, Pagnin D, et al. Prevalence and burden of bipolar disorders in European countries. Eur Neuropsychopharmacol 2005;15:425-34.

15. Allen MH, Currier GW, Hughes DH, et al. The Expert Consensus Guideline Series. Treatment of behavioral emergencies. Postgrad Med 2001:1-88.

16. Breier A, Meehan K, Birkett M, et al. A double-blind, placebocontrolled dose-response comparison of intramuscular olanzapine and haloperidol in the treatment of acute agitation in schizophrenia. Arch Gen Psychiatry 2002;59:441-8.

17. Tran-Johnson TK, Sack DA, Marcus RN, et al. Efficacy and safety of intramuscular aripiprazole in patients with acute agitation: a randomized, double-blind, placebo-controlled trial. J Clin Psychiatry 2007;68:111-9.

18. Heel RC, Brogden RN, Speight TM, et al. Loxapine: a review of its pharmacological properties and therapeutic efficacy as an antipsychotic agent. Drugs 1978;15:198-217.

19. Chakrabarti $A$, Bagnall $A$, Chue $P$, et al. Loxapine for schizophrenia. Cochrane Database Syst Rev 2007;4:CD001943.

20. Bourdinaud V, Pochard F. [Survey of management methods for patients in a state of agitation at admission and emergency departments in France]. Encephale 2003;29:89-98.

21. ADASUVE®. Summary of product characteristics. http://www. ema.europa.eu/docs/en_GB/document_library/EPAR_-_Product Information/human/002400/WC500139409.pdf.

22. Allen MH, Feifel D, Lesem MD, et al. Efficacy and safety of loxapine for inhalation in the treatment of agitation in patients with schizophrenia: a randomized, double-blind, placebo-controlled trial. J Clin Psychiatry 2011;72:1313-21.

23. Lesem MD, Tran-Johnson TK, Riesenberg RA, et al. Rapid acute treatment of agitation in individuals with schizophrenia: multicentre, randomised, placebo-controlled study of inhaled loxapine. $\mathrm{Br} \mathrm{J}$ Psychiatry 2011;198:51-8.

24. Kwentus J, Riesenberg RA, Marandi M, et al. Rapid acute treatment of agitation in patients with bipolar I disorder: a multicenter, randomized, placebo-controlled clinical trial with inhaled loxapine. Bipolar Disord 2012:14:31-40.

25. Wong JG, Clare CH, Holland AJ, et al. The capacity of people with a 'mental disability' to make a health care decision. Psychol Med 2000;30:295-306.

26. Guy W. ECDEU Assessment Manual for Psychopharmacology. Rockville, MD, USA: U.S. Department of Health, Education, and Welfare, 1976.

27. Busner J, Targum SD. The clinical global impressions scale. Psychiatry 2007;4:28-37.

28. ADASUVE $囚$. Highlights of prescribing information. http://www. adasuve.com/PDF/AdasuvePI.pdf.

29. Cohen J. A Coefficient of agreement for nominal scales. Educ Psychol Meas 1960;20:37-46. 